\title{
BENEFÍCIOS DA VERTICALIZAÇÃo dO PARTO
}

\author{
Cláudia Isabel Taborda Amaro \\ Instituto Politécnico de Viseu, Escola Superior de Saúde, Viseu, Portugal \\ essv6093@essv.ipv.pt \\ Hélia Dias \\ UIIPS Instituto Politécnico de Santarém, Escola Superior de Saúde, Santarém, Portugal \\ helia.dias@essaude.ipsantarem.pt \\ Maria José de Oliveira Santos \\ UICISA: E Universidade de Trás-os-Montes e Alto Douro, Escola Superior de Saúde, Vila Real, Portugal \\ mjsantos@utad.pt \\ Paula Alexandra de Andrade Batista Nelas \\ UICISA: E Instituto Politécnico de Viseu, Escola Superior de Saúde, Viseu, Portugal \\ pnelas@gmail.com \\ Emília de Carvalho Coutinho \\ UICISA: E Instituto Politécnico de Viseu, Escola Superior de Saúde, Viseu, Portugal \\ ecoutinhoessv@gmail.com
}

Recepción Artículo: 20 mayo 2021 Admisión Evaluación: 20 mayo 2021 Informe Evaluador 1: 22 mayo 2021 Informe Evaluador 2: 28 mayo 2021 Aprobación Publicación: 01 junio 2021

\section{RESUMO}

São vários os achados históricos que confirmam que o parto, na antiguidade, era realizado em posição vertical. Contudo, com o passar do tempo, aspetos sociais, científicos, culturais e religiosos foram modificando a forma vertical de parir. Aos poucos, as cadeiras de parto foram caindo em desuso, dando lugar ao parto na posição horizontal. No entanto, tem-se vindo a comprovar que a verticalização do parto, tem múltiplas vantagens que fragilizam a defesa de uma prática de parto na posição horizontal. Nesse sentido, pretendeu-se com este estudo, identificar os benefícios da posição vertical no trabalho de parto. A opção metodológica foi a revisão integrativa da literatura, de artigos publicados no friso temporal de 2017-2020, em Português e Inglês disponíveis nas bases de dados Medline $(n=236)$, CINAHL $(n=163)$ e B-0n $(n=244)$, seguindo a estratégia PICOD. Em dezembro de 2020, fez-se a consulta às bases de dados, utilizando a expressão de pesquisa booleana "Delivery, Obstetric"[Mesh]) AND "Patient Positioning"[Mesh] OR ("Delivery, Obstetric"[Mesh] AND upright) OR (upright labor positioning) OR (Vertical[Title] OR upright[Title]) AND (childbirth[Title] OR labor[Title]). Foram critérios de inclusão artigos em texto integral, de acesso livre, estudos qualitativos ou quantitativos, estudos randomizados controlados (RCT), revisões e ensaios clínicos. Não foram consideradas publicações de resumos simples, comunicações em conferências ou artigos de revista sem avaliação por pares. Com base no método de pesquisa foram selecionados 10 artigos, dos quais emergiram os seguintes resultados principais: a verticalização do parto tem 


\section{BENEFíCIOS DA VERTICALIZAÇÃo dO PARTO}

benefícios provocados pela ação da gravidade, favorece o bem-estar fetal, melhora a dinâmica uterina e a estática fetal, diminui a duração do trabalho de parto, diminui a incidência de episiotomia, aumenta os diâmetros pélvicos e proporciona benefícios psicoafectivos à parturiente.Com base nos diferentes estudos, conclui-se assim que parir na posição vertical beneficia a parturiente e o feto devido a fatores fisiológicos e biomecânicos.

Palavras-chave: verticalização do parto; posição vertical; trabalho de parto

\section{ABSTRACT}

Benefits of vertical labor. There are several historical findings that confirm that childbirth, in antiquity, was performed in an upright position. However, over time, social, scientific, cultural and religious aspects have changed the vertical way of giving birth. Gradually, the birth chairs fell into disuse, giving way to the birth in a horizontal position. However, it has been proven that the verticalization of childbirth has in itself intrinsic advantages that increasingly weaken the practice of childbirth in a horizontal position. As methodology, an integrative literature review of articles published in the years 2017-2020, in Portuguese and English, available in the Medline $(n=236)$, CINAHL $(n=163)$ and B-On $(n=244)$ databases, following the PICOD strategy. In December 2020, the survey was conducted using the Boolean search term "Delivery, Obstetric" [Mesh]) AND "Patient Positioning" [Mesh] OR ("Delivery, Obstetric" [Mesh] AND upright) OR (upright labor positioning) OR (Vertical [Title] OR upright [Title]) AND (childbirth [Title] OR labor [Title]), in full text and open access, of qualitative, quantitative, randomized controlled studies (RCT), reviews and clinical trials (Inclusion criteria). Research that resulted in only abstracts, conference presentations or magazine articles without peer review was not considered. Based on the research method, 10 articles were selected, where the following main results emerged: verticalization of childbirth has benefits caused by the action of gravity, favors fetal well-being, improves uterine dynamics and fetal statics, decreases the duration of labor, decreases the incidence of episiotomy, increases pelvic diameters and provides psycho-affective benefits to the parturient woman. Based on the different studies, it is concluded that giving birth in an upright position benefits the parturient and the fetus due to physiological and biomechanical factors.

Keywords: verticalization of childbirth; vertical position; labor

\section{INTRODUÇÃO}

Durante muitos séculos, as mulheres movimentaram-se livremente durante o trabalho de parto. A liberdade de movimento, durante todos os períodos do trabalho de parto, capacita a parturiente para se adaptar à posição que Ihe for mais confortável, permitindo-Ihe optar por várias posições que atualmente são pouco praticadas em contexto clínico. Tradicionalmente, as posições preferencialmente adotadas no segundo período do trabalho de parto eram as posições laterais e verticais. De entre as posições verticais utilizadas a parturiente optava por realizar o período expulsivo em pé, sentada, semi-sentada, de cócoras e ajoelhada, usando as mãos e os joelhos como apoio (Sousa et al., 2018; DiFranco \& Curl, 2014; Miquelutti, Cecatti, Morais, \& Makuch, 2009). Estas posições eram práticas comuns de parto e geralmente ocorriam no domicílio (Musie, Peu, \& Bhana-Pema, 2019).

0 parto, historicamente assistido apenas por mulheres era vedado aos olhares masculinos. Relatos do nascimento de Luís XV descrevem que o seu pai Luís XIV, rei de França, determinou que a sua mãe tinha que parir deitada para que ele, escondido, pudesse observar melhor o nascimento do seu filho, e que, a partir daí, esse método se espalhou pela Europa e depois pelo mundo (Ayres, 2018). Facto é que desde o século XVII que a posição de litotomia se tornou a eleita e habitual na assistência ao parto, uma vez que progressivamente os médicos obstetras procuravam formas de ter maior acessibilidade e visibilidade durante 0 trabalho de parto. Além do referido, naquela época também se tornou conveniente realizar procedimentos invasivos, como episiotomias ou partos com fórceps, para agilizar o trabalho de parto, sobretudo em situações mais complicadas e de complexa resolução (Dundes, 1987). No entanto, existem muitos motivos para não adotar a posição supina ou litotómica durante 0 trabalho de parto (Bick et al., 2017). 
Em países desenvolvidos, a ocorrência do parto em contexto hospitalar provoca na parturiente a limitação da adoção de uma atitude instintiva característica do trabalho de parto, decorrente da medicalização do parto, prática obstétrica e procedimentos protocolados (Bick et al., 2017; Musie et al., 2019).

Na maioria dos hospitais para a analgesia por cateter epidural, hidratação intravenosa, e monitorização contínua da frequência cardíaca fetal e das contrações uterinas, a parturiente deverá permanecer numa posição horizontal, facto que limita, em grande parte, a sua capacidade de mudar ou escolher uma posição diferente. Apesar dos benefícios demonstrados pela adoção do parto vertical, ainda há uma grande percentagem de hospitais que não oferece a opção da verticalização do parto.

Atualmente, as parturientes têm os seus direitos protegidos por diretrizes da Organização Mundial de Saúde (WHO, 2018). Procurando a humanização do parto, estas diretrizes determinam que as mulheres devem ser posicionadas de acordo com a sua escolha e que devem ser incluídas na tomada de decisão sobre os cuidados que recebem. Respeitando a opção da parturiente nas posições escolhidas durante 0 trabalho de parto, os profissionais de saúde devem privilegiar sobretudo as verticais, pelos benefícios que lhes estão inerentes, sendo que a posição de parto em litotomia deve ser evitada no primeiro e segundo período do trabalho de parto (Huang, Zang, Ren, Li, \& Lu, 2019; Musie et al., 2019).

Considera-se posição vertical, todas as posições que proporcionam um ângulo superior a $45^{\circ}$ entre o tronco e os membros inferiores da mulher. A posiç̧ão horizontal inclui a posição supina, de litotomia e semi-reclinada, posições cujo ângulo é inferior a 45 (Mineiro et al., 2016).

Os partos realizados em posições verticais apresentam diminuição do período expulsivo e de scores de dor, quando comparados com partos ocorridos em litotomia. Assumir posições verticais também aumenta a eficiência e a participação ativa da parturiente no trabalho de parto. Os esforços expulsivos são mais eficazes em posições verticais, diminuindo as taxas de parto assistido, duração do período expulsivo e episiotomia (Nilsen et al., 2011).

As posições verticais por beneficiarem da força da gravidade são as mais naturais, tornam as contrações uterinas mais eficazes, favorecem um melhor alinhamento do feto no canal de parto, favorecem a descida e expulsão do feto, e aumentam os diâmetros de saída da pelve, reduzindo complicações maternas e neonatais intraparto (Sousa et al., 2018; DiFranco \& Curl, 2014; Gizzo et al., 2014; Miquelutti et al., 2009).

Hoje em dia, a vivência do trabalho de parto ocorre maioritariamente em contexto hospitalar, onde a prática do parto vertical ainda é considerada como algo invulgar, inovador, arrojado e fora da esfera de controlo dos profissionais de saúde. É consensual que, a posição horizontal dominou os Blocos de Partos, e tornou-se a escoIhida pelas equipas de saúde na assistência ao parto.

Neste sentido, desde a década de 90 do século passado que a OMS emitiu orientações, relativas às práticas adotadas no trabalho de parto e parto, que reforçam a posição dorsal como uma prática prejudicial, enfatizando a adoção de posições verticais como uma prática que deve ser estimulada (OMS, 1996).

Para que a implementação e a adoção da posição vertical pela parturiente, se torne uma preferência, é necessário determinar os benefícios do parto verticalizado, divulgá-los e consciencializar, as mulheres e os profissionais de saúde, sobre os mesmos. É com base nestes pressupostos que se definiu a questão de investigação deste trabalho: "Quais os benefícios da utilização de um parto verticalizado para as mulheres em trabalho de parto?", com o objetivo de identificar os benefícios da posiç̧ão vertical no trabalho de parto.

\section{OBJETIVO DA INVESTIGAÇ̃̃O}

Identificar os benefícios da posição vertical no trabalho de parto.

\section{METODOLOGIA}

A metodologia utilizada neste trabalho foi a revisão integrativa da literatura. Botelho, Cunha \& Macedo (2011) realçam que o método da revisão integrativa possibilita a capacidade de sistematização do conhecimento científico. Defendem que este método deve ser escolhido quando se quer realizar "a síntese e análise do conhecimento científico já produzido sobre o tema investigado" e/ou quando se pretende obter "informações que pos- 
sibilitem aos leitores avaliarem a pertinência dos procedimentos empregados na elaboração da revisão" (Botelho, Cunha \& Macedo, 2011, p.133).

Tendo por base as seis etapas, descritas por Botelho, Cunha \& Macedo (2011, p.129), foi realizada na primeira etapa, a identificação do tema e seleção da questão de pesquisa; na segunda etapa, o estabelecimento dos critérios de inclusão e exclusão; na terceira etapa, a identificação dos estudos pré-selecionados e selecionados; na quarta etapa, a categorização dos estudos selecionados; na quinta etapa, a análise e interpretação dos resultados; e por último na sexta etapa, a apresentação da revisão/síntese do conhecimento.

Após a definição do objetivo deste trabalho foram validados os termos de pesquisa e os critérios de inclusão de pesquisa. As bases de dados selecionadas foram a B-ON, Medline e CINAHL complete através da plataforma EBSCOhost. A pesquisa nas bases de dados, ocorreu no dia 17 de dezembro, utilizando a expressão de pesquisa booleana "Delivery, Obstetric"[Mesh]) AND "Patient Positioning"[Mesh] OR ("Delivery, Obstetric"[Mesh] AND upright) OR (upright labor positioning) OR (Vertical[Title] OR upright[Title]) AND (childbirth[Title] OR labor[Title]), limitada no friso temporal entre 2017 e 2020, aos idiomas Inglês e Português, e com texto integral disponível de estudos qualitativos e quantitativos, estudos randomizados controlados (RCT), revisões e ensaios clínicos. Como critérios de exclusão definiram-se publicações de resumos simples, comunicações em conferências ou artigos de revista sem avaliação por pares.

A formulação da questão de investigação seguiu a metodologia PICOD. Este formato fornece uma estrutura eficiente para a busca de dados em bases eletrônicas. (Ramalho, 2005 citado por Gião \& Pinhão, 2012, P.74).

Decorrente da questão de investigação "quais os benefícios da utilização de um parto verticalizado para as mulheres em trabalho de parto?" apresenta-se no quadro 1 a estratégia de pesquisa no formato PICOD.

Quadro 1 - Apresentação do método PICOD de investigação por revisão integrativa.

\begin{tabular}{|c|c|l|}
\hline P & Quem foi estudado? & $\begin{array}{l}\text { Parturientes com feto único, em apresentação } \\
\text { cefálica, e parto eutócico }\end{array}$ \\
\hline I & O que foi feito? & Estratégia de verticalização do parto \\
\hline C & $\begin{array}{c}\text { Comparações entre } \\
\text { resultados }\end{array}$ & $\begin{array}{l}\text { Comparação com as mesmas participantes que } \\
\text { tiveram um parto em posição supina ou em } \\
\text { litotomia }\end{array}$ \\
\hline O & $\begin{array}{c}\text { Quais foram os resultados } \\
\text { ou efeitos? }\end{array}$ & $\begin{array}{l}\text { Parto eutócico com baixas lesões neonatais e } \\
\text { maternas e com menor duração do segundo } \\
\text { período do trabalho de parto }\end{array}$ \\
\hline D & $\begin{array}{c}\text { Desenho do Estudo } \\
\text { Como é? }\end{array}$ & $\begin{array}{l}\text { Estudos do tipo qualitativo, quantitativo ou } \\
\text { mistos, publicados integralmente em Inglês e } \\
\text { Português }\end{array}$ \\
\hline & $\begin{array}{l}\text { Expressão de pesquisa booleana: "Delivery, Obstetric"[Mesh]) AND } \\
\text { "Patient Positioning"[Mesh] OR ("Delivery, Obstetric"[Mesh] AND upright) } \\
\text { OR (upright labor positioning) OR (Vertical[Title] OR upright[Title]) AND } \\
\text { (childbirth[Title] OR labor[Title]) }\end{array}$ \\
\hline
\end{tabular}

Utilizando a expressão de pesquisa booleana atrás referida, na base de dados CINAHL complete obtiveramse 163 artigos, na B-ON obtiveram-se 244 artigos e na base de dados MEDLINE obtiveram-se 236 artigos (total de 643 resultados), conforme figura 1.

Encontraram-se 185 artigos duplicados, pelo que foram considerados elegíveis 458 artigos. Após leitura pelo título e resumo foram excluídos 326 artigos por não estarem enquadrados na temática em revisão e na resposta à questão definida, 26 por não cumprirem os critérios de inclusão (idioma e texto integral) e artigos que apesar de terem interesse pelo título ou resumo, apenas possuíam o resumo (por se tratarem de trabalhos de apresentação de conferências, posters ou sessões) e por estarem apresentadas em revistas sem revisão por pares (excluídos pelos critérios de exclusão 96 pesquisas). 
Restaram 10 artigos os quais constituíram o corpo de análise desta revisão e permitiram dar resposta à questão de investigação inicialmente formulada.

Figura 1 - Diagrama de obtenção de resultados usando as bases de dados e critérios adotados nesta revisão integrativa. Prisma 2009 adaptado de JBI (Peters et al., 2020).

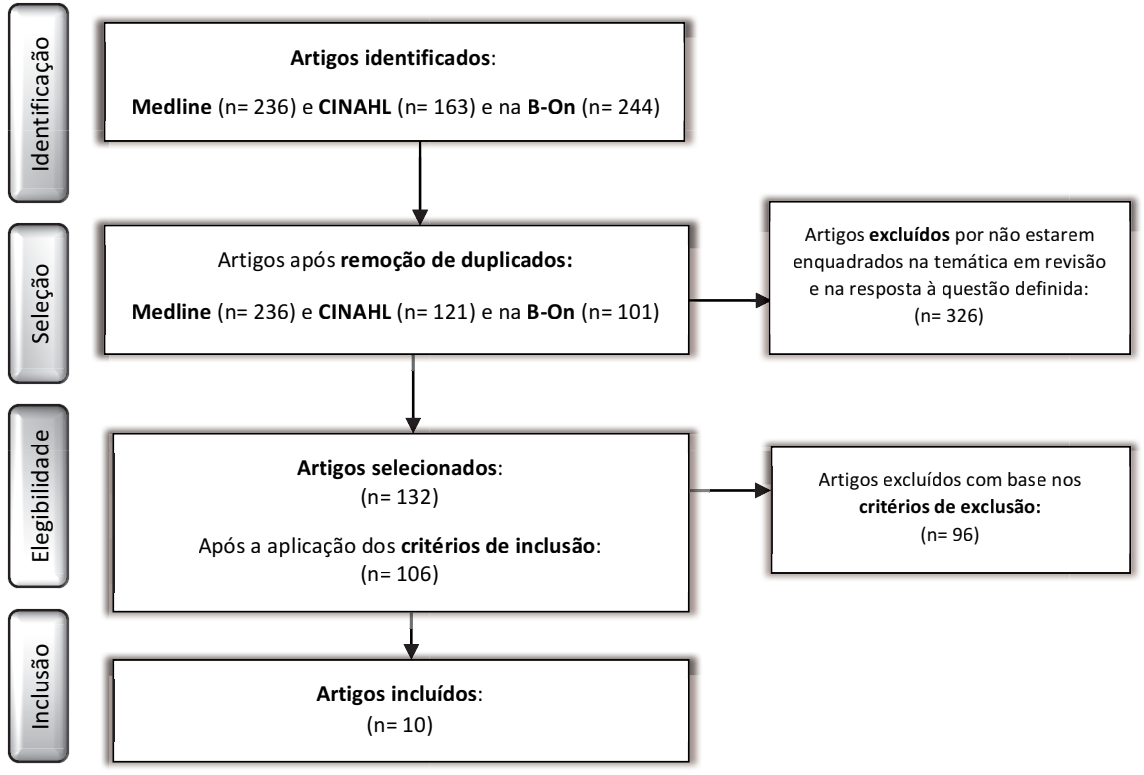

\section{RESULTADOS ALCANÇADOS}

Na triagem dos artigos extraídos, de acordo com as fases do prisma (apresentadas na figura 1), resultaram dez estudos relevantes para o desenvolvimento desta revisão integrativa da literatura. Cada um dos estudos, com métodos de investigação distintos, permitiu responder à questão de investigação. Os mesmos são apresentados no quadro seguinte.

\section{Quadro 2 - Resultados obtidos recorrendo ao método PICOD}

\begin{tabular}{|c|c|c|c|}
\hline Título/Referência & Objetivos & Métodos & Resultados/Conclusões \\
\hline $\begin{array}{l}\text { 1. EFFECTS OF UPRIGHT } \\
\text { POSITIONS DURING THE } \\
\text { SECOND STAGE OF LABOUR } \\
\text { FOR WOMEN WITHOUT } \\
\text { EPIDURAL ANALGESIA: A } \\
\text { META-ANALYSIS } \\
\text { (Zang et al., 2020) } \\
\text { (Base de dados: B-on) }\end{array}$ & $\begin{array}{l}\text { Avaliar os efeitos das } \\
\text { posições verticais sobre os } \\
\text { resultados maternos para as } \\
\text { mulheres sem epidural, em } \\
\text { comparação com as posições } \\
\text { supinas durante o segundo } \\
\text { período do trabalho de parto. }\end{array}$ & $\begin{array}{l}\text { Meta-análise de ensaios clínicos } \\
\text { randomizados usando o Review } \\
\text { Manager 5.3.; } \\
\text { Revisão de literatura nas bases de } \\
\text { dados Pubmed, Embase, Cochrane } \\
\text { library, CINAHL e ProQuest; } \\
\text { Participantes: mulheres com } \\
\text { gravidez de baixo risco, entre } 37- \\
42 \text { semanas de gravidez, com um } \\
\text { único feto de apresentação cefálica } \\
\text { e sem analgesia epidural. }\end{array}$ & $\begin{array}{l}\text { Identificação de } 6017 \text { estudos que } \\
\text { depois de removidos os duplicados } \\
\text { resultaram } 4103 \text { estudos. No geral, } \\
\text { foram realizados } 12 \text { estudos incluindo } \\
4.314 \text { mulheres; } \\
\text { A adoção de posições verticais diminui } \\
\text { significativamente as lesões perineais, } \\
\text { encurta a duração do segundo período } \\
\text { do trabalho de parto e do uso de } \\
\text { instrumentos; } \\
\text { As posições verticais aumentam os } \\
\text { diâmetros transversais e ântero- } \\
\text { posteriores da pelve e as contrações } \\
\text { uterinas, quando comparadas com } \\
\text { posições supinas, sendo por isso muito } \\
\text { mais benéficas tanto para a parturiente } \\
\text { como para o feto. }\end{array}$ \\
\hline
\end{tabular}




\begin{tabular}{|c|c|c|c|}
\hline 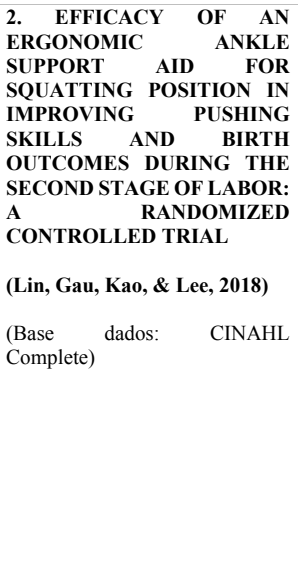 & $\begin{array}{l}\text { Explorar os efeitos do parto } \\
\text { e das posições verticais } \\
\text { adotadas (semi-inclinada, } \\
\text { agachada e agachada com } \\
\text { apoios nos tornozelos) de } \\
\text { três grupos de mulheres, } \\
\text { durante a segunda fase do } \\
\text { trabalho de parto. }\end{array}$ & $\begin{array}{l}\text { Estudo controlado randomizado, } \\
\text { não cego; } \\
\text { As participantes de } 38-42 \text { semanas } \\
\text { de gestação, com idade > a } 18 \text { anos } \\
\text { e sem complicações médicas foram } \\
\text { designadas aleatoriamente para } \\
\text { cada um dos grupos que } \\
\text { correspondiam a uma posição: } \\
\text { agachada com apoio de tornozelos, } \\
\text { sem apoio e semi-inclinada; } \\
\text { Critérios de exclusão: obesidade, } \\
\text { analgesia epidural, emergências } \\
\text { obstétricas e cesarianas. }\end{array}$ & $\begin{array}{l}\text { Participantes que usaram a posição de } \\
\text { agachamento (grupo experimental e } \\
\text { grupo de comparação A) tiveram } \\
\text { tempos médios de parto } \\
\text { significativamente mais baixos (18 a } 25 \\
\text { minutos) do que as participantes que } \\
\text { adotaram uma posição semi-inclinada, } \\
\text { devido ao maior efeito da força } \\
\text { gravitacional; } \\
\text { A posição de agachamento com apoio } \\
\text { aumenta significativamente a eficácia, } \\
\text { no entanto não existe diferença da dor } \\
\text { sentida entre as três posições; } \\
70 \% \text { das participantes não conseguiu } \\
\text { manter inteiramente a posição de } \\
\text { agachamento sem apoio nos tornozelos } \\
\text { tendo de alternar entre esta e a de semi- } \\
\text { inclinada, de modo a aliviar as pernas; } \\
\text { Os autores consideraram que são } \\
\text { necessários mais estudos para analisar a } \\
\text { eficácia das posições verticais na dor } \\
\text { sentida durante o trabalho de parto, em } \\
\text { comparação com posições supinas. }\end{array}$ \\
\hline $\begin{array}{l}\text { 3. DOES BREECH DELIVERY } \\
\text { IN AN UPRIGHT POSITION } \\
\text { INSTEAD OF ON THE BACK } \\
\text { IMPROVE OUTCOMES AND } \\
\text { AVOID CESAREANS }\end{array}$ & $\begin{array}{l}\text { Comparação entre as mães } \\
\text { com parto eutócico vertical } \\
\text { (apoio na cama, de joelhos, } \\
\text { de quatro ou de pé), supino e } \\
\text { cesariana. }\end{array}$ & $\begin{array}{l}\text { Estudo de coorte retrospetivo } \\
\text { realizado num centro em Frankfurt } \\
\text { (Alemanha), com todas as } \\
\text { mulheres em parto pélvico de um } \\
\text { único feto com }>37 \text { semanas } \\
\text { gestação, entre janeiro de } 2004 \text { e } \\
\text { junho de } 2011 \text {. }\end{array}$ & $\begin{array}{l}\text { Das } 269 \text { grávidas que tiveram um parto } \\
\text { eutócico bem-sucedido, a maioria } \\
\text { adotou posições verticais, e tiveram um } \\
\text { segundo período de trabalho de parto } \\
\text { muito inferior em comparação com } \\
\text { partos supinos ou em litotomia; }\end{array}$ \\
\hline $\begin{array}{l}\text { (Louwen, Daviss, Johnson, \& } \\
\text { Reitter, 2017) } \\
\text { (Base de dados: B-on) }\end{array}$ & & & $\begin{array}{l}\text { Quando a posição vertical foi usada } \\
\text { quase exclusivamente, a taxa de } \\
\text { cesarianas diminuiu; O parto pélvico } \\
\text { por via vaginal em posição vertical está } \\
\text { também associado a menor frequência } \\
\text { de lesões e manipulações para extrair o } \\
\text { feto, quando comparado com o parto } \\
\text { por via vaginal em posição supina ou } \\
\text { litotómica. }\end{array}$ \\
\hline $\begin{array}{l}\text { 4. IS THERE AN IMPACT OF } \\
\text { FEET POSITION } \text { ON } \\
\text { SQUATTING BIRTH } \\
\text { POSITION? AN INNOVATIVE } \\
\text { BIOMECHANICAL PILOT } \\
\text { STUDY } \\
\text { (Desseauve, Fradet, Lacouture, } \\
\text { \& Pierre, 2019) } \\
\text { (Base de dados: MEDLINE } \\
\text { Complete) }\end{array}$ & $\begin{array}{l}\text { Comparar o impacto da } \\
\text { postura do pé nos parâmetros } \\
\text { biomecânicos durante uma } \\
\text { posição de parto agachada: } \\
\text { na ponta dos pés vs pés } \\
\text { apoiados no chão. }\end{array}$ & $\begin{array}{l}\text { Estudo piloto com treze mulheres } \\
\text { grávidas com mais de } 32 \text { semanas } \\
\text { que não estavam em trabalho de } \\
\text { parto foram avaliadas na posição } \\
\text { de cócoras, primeiro } \\
\text { espontaneamente e, em segundo } \\
\text { lugar, com a postura do pé que não } \\
\text { foi tomada espontaneamente (na } \\
\text { ponta dos pés vs com os pés } \\
\text { apoiados). Para cada posição, foi } \\
\text { usado o sistema optoelectrónico de } \\
\text { captura de movimento; } \\
\text { Participantes: mulheres grávidas } \\
\text { com mais de } 18 \text { anos ou mais } 32 \\
\text { semanas de gestação, com um } \\
\text { índice de massa corporal<40 e sem } \\
\text { doenças inflamatórias articulares } \\
\text { ou hipermobilidade articular, como } \\
\text { a síndrome de Marfan. }\end{array}$ & $\begin{array}{l}\text { Durante o trabalho de parto, usando a } \\
\text { posição de cócoras (que os autores } \\
\text { consideraram ser a posição "natural" do } \\
\text { parto), a postura do pé tem uma função } \\
\text { biomecânica com impacto na curva } \\
\text { lombar e na pelve, o ângulo da curva da } \\
\text { escavação pélvica é menos } \\
\text { evidenciado, ocorre uma retificação do } \\
\text { canal de parto e alinhamento do feto na } \\
\text { bacia materna; } \\
\text { Ao comparar as posições de } \\
\text { agachamento (em ponta dos pés } v s \text { pés } \\
\text { apoiados), a postura de pés apoiados } \\
\text { está mais perto do ideal, apesar da } \\
\text { maioria das participantes não adotar } \\
\text { esta posição espontaneamente. }\end{array}$ \\
\hline
\end{tabular}




\begin{tabular}{|c|c|c|c|}
\hline $\begin{array}{l}\text { 5. A PROSPECTIVE } \\
\text { RANDOMIZED TRIAL OF } \\
\text { POSTURAL CHANGES VS } \\
\text { PASSIVE SUPINE LYING } \\
\text { DURING THE SECOND } \\
\text { STAGE OF LABOR UNDER } \\
\text { EPIDURAL ANALGESIA } \\
\text { (Simarro et al., 2017) } \\
\begin{array}{l}\text { (Base de dados: MEDLINE } \\
\text { complete) }\end{array}\end{array}$ & 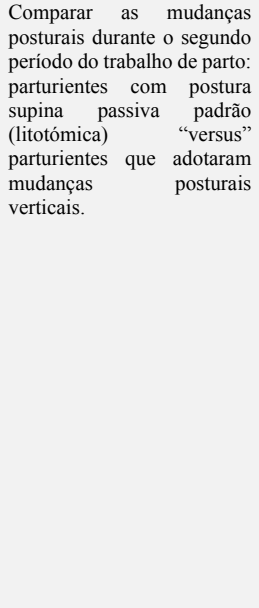 & $\begin{array}{l}\text { Ensaio clínico randomizado que } \\
\text { visa o estudo das mudanças } \\
\text { posturais durante o segundo } \\
\text { período do trabalho de parto, } \\
\text { comparando as parturientes que } \\
\text { assumiram uma postura supina } \\
\text { passiva padrão, com todas as } \\
\text { parturientes do grupo de estudo } \\
\text { que adotaram mudanças posturais. } \\
\text { Todas as parturientes foram } \\
\text { monitorizadas constantemente; } \\
\text { Foram incluídas grávidas entre } 37- \\
42 \text { semanas de gestação, sem } \\
\text { problemas médicos associados e } \\
\text { com parto vaginal espontâneo. } \\
\text { Todas fizeram analgesia epidural; } \\
\text { Os critérios de exclusão incluíram } \\
\text { parturientes com dificuldades em } \\
\text { entender os procedimentos } \\
\text { (barreira linguística), com } \\
\text { cesariana programada, partos } \\
\text { induzidos, ou complicações } \\
\text { obstétricas (hipertensão arterial, } \\
\text { restrição de crescimento intra- } \\
\text { uterino). }\end{array}$ & $\begin{array}{l}\text { O estudo mostra que a adoção } \\
\text { sistemática de mudanças posturais } \\
\text { (dentro das verticalizações) durante o } \\
\text { segundo período do trabalho de parto } \\
\text { está significativamente associado a um } \\
\text { parto mais curto, menos instrumentado, } \\
\text { a uma diminuição da incidência de } \\
\text { cesarianas, a uma menor necessidade de } \\
\text { realizar episiotomias, e a uma menor } \\
\text { ocorrência de lacerações perineais de } \\
\text { terceiro grau, em comparação com } \\
\text { partos supinos. }\end{array}$ \\
\hline $\begin{array}{l}\text { 6. PERCEPÇÃO } \\
\text { PUERPERAS SOBRE } \\
\text { POSIÇÃO } \\
\text { VERTICAL NO PARTO } \\
\text { (Sousa et al., 2018) } \\
\begin{array}{l}\text { (Base de dados: MEDLINE } \\
\text { complete) }\end{array}\end{array}$ & $\begin{array}{l}\text { Descrever a perceção de } \\
\text { puérperas acerca da posição } \\
\text { vertical adotada no trabalho } \\
\text { de parto e parto. }\end{array}$ & $\begin{array}{l}\text { Revisão descritiva qualitativa } \\
\text { numa maternidade de referência de } \\
\text { Teresina, Piauí (Brasil), durante o } \\
\text { ano de 2014; } \\
\text { Critérios de inclusão: puérperas } \\
\text { que tenham passado pela } \\
\text { experiência do parto vertical e } \\
\text { possuam histórico de parto } \\
\text { eutócico na posição horizontal, } \\
\text { estar internada na maternidade e } \\
\text { ser maior de idade; } \\
\text { Critérios de exclusão: puérperas } \\
\text { com fetos múltiplos, nado-morto } \\
\text { ou com deficiências auditivas; } \\
\text { Método de obtenção de dados por } \\
\text { entrevista (15 minutos cada) com } \\
\text { questões estruturadas sobre dados } \\
\text { demográficos e antecedentes } \\
\text { obstétricos (tais como o número de } \\
\text { gestações prévias, paridade, } \\
\text { número de consultas pré-natais e } \\
\text { posições utilizadas em partos } \\
\text { anteriores e no atual), além das } \\
\text { questões de pesquisa: "Como foi } \\
\text { seu recente parto?", "Você sabia } \\
\text { que existem diferentes posições } \\
\text { para a mulher parir?", "O que você } \\
\text { tem a dizer sobre a posição } \\
\text { vertical?"; }\end{array}$ & $\begin{array}{l}\text { Entrevistas realizadas a } 8 \text { grávidas com } \\
\text { idades entre } 18-33 \text { anos que já tinham } \\
\text { adotado posições verticais: } 2 \text { de pé, } 1 \\
\text { sentada no cavalinho e } 5 \text { sentadas num } \\
\text { banco; } \\
\text { A pesquisa fez surgir quatro categorias: } \\
\text { o tipo de conhecimento sobre as } \\
\text { posições verticais, a perceção da } \\
\text { presença da enfermeira obstetra com o } \\
\text { incentivo às posições verticais, as } \\
\text { recordações da vivência de partos em } \\
\text { outras posições e a perceção das } \\
\text { puérperas sobre o parto na posição } \\
\text { vertical; } \\
\text { Observada uma lacuna no } \\
\text { conhecimento de diferentes posições de } \\
\text { parto possíveis, não sendo apresentadas } \\
\text { durante as consultas pré-natais, as mães } \\
\text { que adotaram alguma das posições } \\
\text { verticais, apenas se aperceberam de que } \\
\text { seria vantajosa, no momento do parto; } \\
\text { As enfermeiras obstetras foram } \\
\text { reconhecidas como o elo principal na } \\
\text { confiança da parturiente e no à vontade } \\
\text { na mudaça da posição horizontal para } \\
\text { a vertical, na qual a parturiente se } \\
\text { sentiria mais confortável; } \\
\text { As puérperas avaliaram positivamente a } \\
\text { posição vertical. Concluíram que as } \\
\text { posições verticais que acabaram por }\end{array}$ \\
\hline & & $\begin{array}{l}\text { Os dados foram analisados por } \\
\text { meio da técnica de análise de } \\
\text { conteúdo. }\end{array}$ & $\begin{array}{l}\text { adotar foram mais confortáveis, } \\
\text { proporcionaram uma descida mais } \\
\text { rápida do feto, uma redução do tempo } \\
\text { do trabalho de parto, uma diminuição da } \\
\text { dor, maior autonomia e menor } \\
\text { intervenção dos profissionais de saúde, } \\
\text { apresentado assim vários beneficios. }\end{array}$ \\
\hline
\end{tabular}




\begin{tabular}{|c|c|c|c|}
\hline $\begin{array}{l}\text { 7. POSITION IN THE SECOND } \\
\text { STAGE OF LABOUR FOR } \\
\text { WOMEN } \\
\text { EPIDURAL } \text { WITHOUT } \\
\text { (REVIEW) } \\
\begin{array}{l}\text { (Gupta, Sood, Hofmeyr, \& } \\
\text { Vogel, 2017) } \\
\text { (Base de dados: MEDLINE } \\
\text { complete) }\end{array}\end{array}$ & $\begin{array}{l}\text { Determinar os possíveis } \\
\text { benefícios e riscos do uso de } \\
\text { diferentes posições de parto } \\
\text { durante o segundo período } \\
\text { do trabalho de parto, sem } \\
\text { analgesia epidural, nos } \\
\text { resultados maternos, fetais e } \\
\text { neonatais. }\end{array}$ & $\begin{array}{l}\text { Pesquisa no Cochrane Pregnancy } \\
\text { and Childbirth's Trials Register } \\
\text { (efetuada a } 30 \text { de novembro de } \\
\text { 2016) e em listas de referências de } \\
\text { estudos recuperados; } \\
\text { Critérios de inclusão: ensaios } \\
\text { clínicos randomizados, quasi- } \\
\text { randomizados ou randomizados } \\
\text { por cluster, de qualquer posição } \\
\text { vertical assumida por mulheres } \\
\text { grávidas durante o segundo } \\
\text { período do trabalho de parto, em } \\
\text { comparação com posições supinas. } \\
\text { As comparaços secundárias } \\
\text { incluem a comparação das } \\
\text { posições verticais e supinas; } \\
\text { A recolha e análise de dados foi } \\
\text { realizada por dois revisores que } \\
\text { avaliaram independentemente os } \\
\text { ensaios para inclusão e avaliaram a } \\
\text { sua qualidade. Pelo menos dois } \\
\text { autores da revisão extraíram os } \\
\text { dados, tendo sido verificados } \\
\text { quanto à sua precisão; } \\
\text { A qualidade e importação dos } \\
\text { dados foi avaliada usando a } \\
\text { abordagem GRADE (GRADEpro } \\
\text { Guideline Development Tool). }\end{array}$ & $\begin{array}{l}\text { Os resultados desta revisão sugerem } \\
\text { vários benefícios das posições verticais } \\
\text { em parturientes sem analgesia epidural, } \\
\text { tais como a redução da duração do } \\
\text { segundo período do trabalho de parto, } \\
\text { redução nas taxas de realização de } \\
\text { episiotomia e parto assistido (com o uso } \\
\text { de fórceps e ventosa); } \\
\text { Não foi observada nenhuma diferença } \\
\text { clara na duração do segundo período do } \\
\text { trabalho de parto entre as diferentes } \\
\text { posições verticais adotadas (uso de } \\
\text { cadeira, banco de parto ou de cócoras); } \\
\text { No entanto, é necessário reconhecer que } \\
\text { existem alguns riscos, tais como a maior } \\
\text { perda de sangue (superior a } 500 \mathrm{ml} \text { ) e } \\
\text { de lesões de segundo grau. }\end{array}$ \\
\hline $\begin{array}{l}\text { 8. UPRIGHT VERSUS LYING } \\
\text { DOWN POSITION IN SECOND } \\
\text { STAGE OF LABOUR IN } \\
\text { NULLIPAROUS WOMEN } \\
\text { WITH LOW DOSE EPIDURAL: } \\
\text { BUMPES RANDOMISED } \\
\text { CONTROLLED TRIAL } \\
\text { (Brocklehurst, 2017) } \\
\text { (Base de dados: CINAHL } \\
\text { Complete) }\end{array}$ & $\begin{array}{l}\text { Determinar se a } \\
\text { verticalização no segundo } \\
\text { período do trabalho de parto } \\
\text { em mulheres nulíparas e com } \\
\text { uma dose baixa de epidural, } \\
\text { aumenta a incidência de } \\
\text { parto vaginal espontâneo, } \\
\text { comparativamente } \\
\text { posições horizontais. }\end{array}$ & $\begin{array}{l}\text { Ensaio clínico multicentralizado, } \\
\text { pragmático, randomizado e } \\
\text { controlado individualmente, } \\
\text { realizado em } 41 \text { unidades de parto } \\
\text { em hospitais do Reino Unido, } \\
\text { desde } 04 / 10 / 2010 \text { até } 31 / 01 / 2014 \text {; } \\
\text { Inclusão: } 3093 \text { participantes do } \\
\text { género feminino, com mais de } 16 \\
\text { anos de idade, mais de } 37 \text { semanas } \\
\text { de gestação, no segundo período do } \\
\text { trabalho de parto, com um único } \\
\text { feto de apresentação cefálica e com } \\
\text { uma dose baixa de analgesia } \\
\text { epidural; } \\
\text { Intervenção: mulheres alocadas na } \\
\text { posição que mantivesse a pelve no } \\
\text { plano mais vertical possível, } \\
\text { durante o segundo período do } \\
\text { trabalho de parto de preferência até } \\
\text { ao nascimento (incluindo } \\
\text { caminhar, levantar, sentar fora da } \\
\text { cama, ajoelhar apoiada ou apoiada } \\
\text { na posição vertical); ou deitada } \\
\text { (lateral direita ou esquerda, para } \\
\text { evitar a compressão da veia } \\
\text { cava inferior ou da aorta, com } \\
\text { inclinação de } 30^{\circ} \text { da cama), de } \\
\text { modo a, neste caso, manter a pelve } \\
\text { no plano horizontal o maior tempo } \\
\text { possível durante o segundo período } \\
\text { do trabalho de parto até à } \\
\text { finalização do trabalho de parto; } \\
\text { Como este estudo foi pragmático, } \\
\text { houveram razões inevitáveis para } \\
\text { mudar a posição materna, como } \\
\text { por exemplo, sofrimento fetal ou } \\
\text { para ajudar a melhorar o puxo no } \\
\text { período expulsivo. Assim as } \\
\text { mulheres eram livres para mudar } \\
\text { de posição se assim o desejassem } \\
\text { em qualquer fase do trabalho de } \\
\text { parto; }\end{array}$ & $\begin{array}{l}\text { A posição materna no segundo período } \\
\text { do trabalho de parto pode afetar a } \\
\text { incidência de parto vaginal espontâneo; } \\
\text { É possível que as mulheres na posição } \\
\text { vertical, devido à epidural, adquiram } \\
\text { um bloqueio mais denso ao redor do } \\
\text { canal de parto resultante dos efeitos } \\
\text { potenciais da postura e do nível de } \\
\text { absorção dos fármacos no espaço } \\
\text { epidural, o que pode ter tornado os } \\
\text { esforços expulsivos menos eficazes; } \\
\text { O estudo sugere que as mulheres do } \\
\text { grupo das posições verticais, que } \\
\text { possam ter estado sentadas, podem ter } \\
\text { uma saída pélvica restrita por causa da } \\
\text { pressão no cóccix ou por causa da } \\
\text { obstrução venosa, causando edema do } \\
\text { trato genital inferior e obstrução dos } \\
\text { tecidos moles da saída pélvica; } \\
\text { Em mulheres nulíparas em trabalho de } \\
\text { parto a termo com epidural e um feto } \\
\text { único, a política de adotar a posição } \\
\text { horizontal no segundo período do } \\
\text { trabalho de parto aumenta as chances de } \\
\text { parto vaginal espontâneo. Apesar desta } \\
\text { evidência não ter sido medida, poderá } \\
\text { dever-se aos efeitos da analgesia } \\
\text { epidural impossibilitarem a adoção de } \\
\text { posições verticais. }\end{array}$ \\
\hline
\end{tabular}




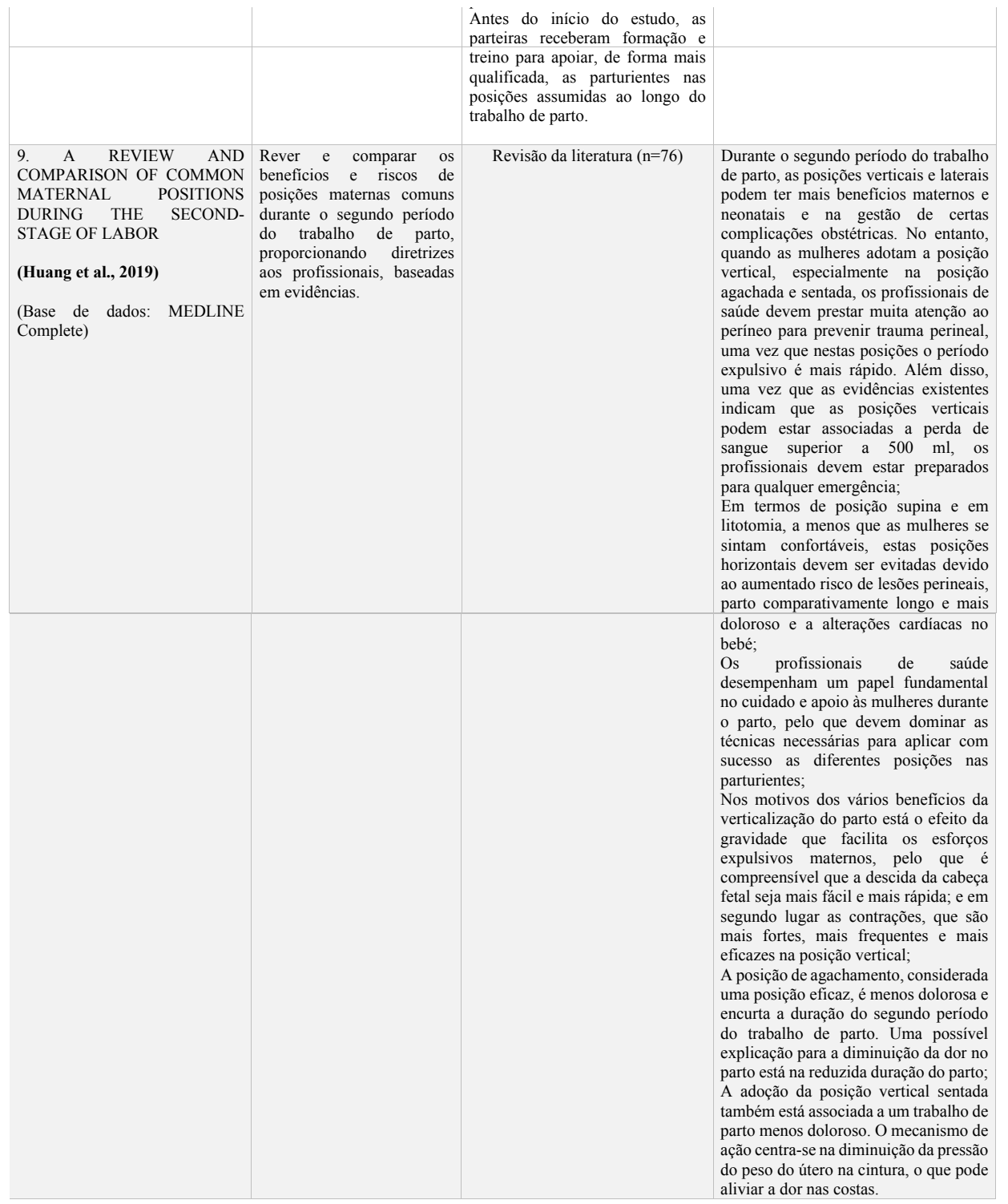




\begin{tabular}{|c|c|c|c|}
\hline $\begin{array}{l}\text { 10. REDUCING PRIMARY } \\
\text { CESAREANS } \\
\text { AN INNOVATIVE } \\
\text { MULTIPRONGED APPROACH } \\
\text { TO } \\
\text { PHYSIOLOGIC } \\
\text { LABOR AND VAGINAL } \\
\text { BIRTH } \\
\text { (Gams, Neerland, \& Kennedy, } \\
\text { 2019) } \\
\text { (Base de dados: MEDLINE } \\
\text { Complete) }\end{array}$ & $\begin{array}{l}\text { Projeto com o objetivo de } \\
\text { reduzir a taxa de cesarianas } \\
\text { em nulíparas, de termo, com } \\
\text { um único feto e de } \\
\text { apresentação cefálica. }\end{array}$ & 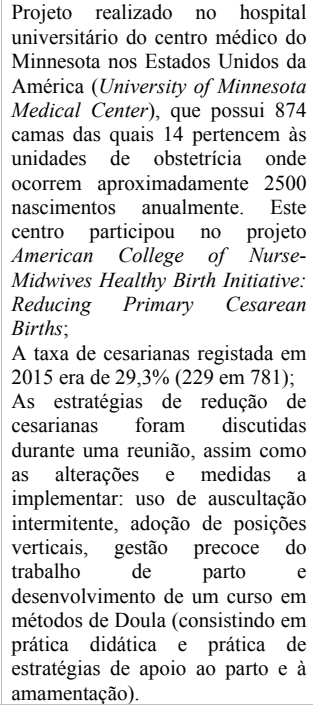 & $\begin{array}{l}\text { Após a implementação do projeto, a } \\
\text { taxa de cesarianas diminuiu para } 26,1 \% \\
\text { em } 2016 \text { e para } 25,3 \% \text { em } 2017 \text {; } \\
\text { A percentagem de grávidas que } \\
\text { adotaram as posições verticais } \\
\text { aumentou de } 13,8 \% \text { para } 29,7 \% \text {; } \\
\text { A duração média da primeira fase do } \\
\text { trabalho de parto para as mulheres que } \\
\text { permaneceram na cama durante a maior } \\
\text { parte do tempo, foi } 2 \text { horas a mais, em } \\
\text { comparação com as mulheres que } \\
\text { adotaram posições verticais por mais de } \\
50 \% \text { do tempo; } \\
\text { A taxa de aumento do trabalho de parto } \\
\text { para mulheres verticalizadas em mais } \\
\text { de } 50 \% \text { do tempo, foi } \\
\text { significativamente menor em relação às } \\
\text { mulheres que estavam em pé menos de } \\
50 \% \text { do tempo ( } 26,3 \% \text { vs } 54,4 \% \text { ); } \\
\text { Permanecer em posição vertical por } \\
\text { mais de } 50 \% \text { do trabalho de parto foi } \\
\text { significativamente associado a não } \\
\text { necessitar de analgesia epidural ( } 7,3 \% \\
v s 78,3 \% \text {; } \\
\text { Passar mais de } 50 \% \text { do tempo de parto } \\
\text { em pé foi significativamente associado } \\
\text { a uma menor probabilidade de parto por } \\
\text { cesariana ( } 4,9 \% v s 18,6 \%) \text {. }\end{array}$ \\
\hline
\end{tabular}

\section{DISCUSSÃO}

Desde 0 momento em que o parto começou com uma assistência medicalizada, inserido em contexto hospitalar, as práticas de cuidados à parturiente foram alteradas, interferindo inevitavelmente nos mecanismos fisiológicos do parto, nomeadamente no posicionamento da mulher (Mineiro et al., 2016). Atualmente, em países desenvolvidos, uma percentagem bastante significativa de grávidas que entra em trabalho de parto num hospital, com assistência por equipa multidisciplinar, é colocada em posição horizontal (Gupta et al., 2017; Lin et al., 2018). Muitos destes hospitais ainda não oferecem a opção de posições verticalizadas, embora vários estudos já tenham demonstrado os seus benefícios (Sousa et al., 2018; Gupta et al., 2017; Huang et al., 2019).

Além da sua naturalidade implícita (devido à força da gravidade), está provado que o parto em posição vertical é mais eficaz do que em posição supina ou em litotomia, em consequência de múltiplos fatores fisiológicos e biomecânicos, que passo a mencionar: a gravidade favorece 0 encravamento, a descida da apresentação e a expulsão do feto; os esforços expulsivos são maximizados pela ação da gravidade; 0 ângulo da curva da escavação pélvica é menos evidenciado, ocorre uma retificação do canal de parto e alinhamento do feto na pelve materna; verifica-se uma melhoria do fluxo uteroplacentário devido à diminuição da compressão, pelo útero, nos grandes vasos maternos (artéria aorta e veia cava); existe melhoria da ventilação materna e do equilíbrio ácido-base materno e fetal; existem menos alterações anormais da frequência cardíaca fetal; os membros inferiores otimizam os esforços expulsivos maternos; diminui a incidência de partos distócicos; existe maior eficiência das contrações uterinas, que se tornam mais frequentes e mais intensas; aumenta os diâmetros ântero-posterior e transverso da pelve (especialmente a posição de cócoras); melhora a estática fetal e diminui a percentagem de posições anómalas da cabeça fetal; há uma menor necessidade de realizar episiotomia e menor ocorrência de lacerações perineais de terceiro grau (em comparação com partos horizontais); o trabalho de parto é vivenciado com níveis de desconforto e de dor (principalmente lombar) menores; e por último, a possibilidade de liberdade de movimentos aumenta os níveis de endorfinas, que levam a uma diminuição dos níveis de dor e a um aumento dos níveis de satisfação da parturiente (DiFranco \& Curl, 2014; Huang et al., 2019; Lin et al., 2018; Louwen et al., 2017; Miquelutti et al., 2009; Mineiro et al., 2016; Nilsen et al., 2011; Zang et al., 2020). 
Para além do referido, na verticalização do parto, existe maior autonomia, participação, controlo e envolvimento da parturiente no trabalho de parto e período expulsivo. Verifica-se maior interação e colaboração com os profissionais de saúde e dá oportunidade à parturiente de ter um parto mais humanizado (Mineiro et al., 2016).

A humanização do parto ocorre quando as parturientes, em posições verticais, ficam mais confortáveis e confiantes no seu potencial para parir, o parto é mais rápido e, por conseguinte, o cansaço é menor, ocorre menos dor, existe maior autonomia e menor intervenção profissional (Sousa et al., 2018).

No entanto, apesar dos benefícios também é necessário ter em conta algumas desvantagens ou situações que podem ocorrer na verticalização do parto, tais como: lesões perineais de segundo grau; esforço excessivo e extenuante nos membros inferiores, joelhos e tornozelos; a probabilidade de uma perda sanguínea maior que $500 \mathrm{ml}$; e na posição sentada, as parturientes nulíparas com analgesia epidural, poderem ter uma saída pélvica restrita por causa da pressão no cóccix ou por obstrução venosa, que causa edema genital inferior e obstrução dos tecidos moles da saída pélvica. Para além do referido, em parturientes que assumam a posição de agachamento ou sentada, o profissional de saúde deve prestar uma maior atenção ao períneo para evitar trauma perineal (Gupta et al., 2017; Huang et al., 2019; Lin et al., 2018).

De entre as posições verticais, a parturiente pode optar por aquela que lhe for mais conveniente e que cause maior bem-estar e menor constrangimento físico, assim como poderá alternar entre elas, podendo deste modo assumir, durante os períodos do trabalho de parto, todas as posições verticais possíveis (Gupta et al., 2017; Simarro et al., 2017).

A posição em pé é altamente ergonómica, pois permite que a ação da gravidade potencialize 0 efeito das contrações e dos puxos maternos, ajudando o feto a descer pela cavidade pélvica. Está associada ao menor risco de lesões do esfíncter anal durante o parto (Zang et al., 2020). Por outro lado, também alivia os vasos uterinos e a veia cava inferior do peso fetal e do útero em contração, o que evita qualquer redução no fluxo sanguíneo materno e isquémia placentária, o que poderia causar sofrimento fetal (Huang et al., 2019). Todas as posições verticais estimulam a frequência das contrações. É, no entanto, inconveniente para o profissional de saúde ver ou auxiliar no processo de parto (Louwen et al., 2017; Zang et al., 2020).

A posição semi-reclinada ganhou popularidade no século XVII, em grande parte devido à influência de Mauiceau, que foi um dos primeiros barbeiros-cirurgiões a envolver-se ativamente na obstetrícia (Huang et al., 2019). Pode ter alguma conexão com o facto de que esse também foi o período do surgimento da litotomia, que exigia essa visão precisa do períneo. Esta posição mantém a mulher visível e confere maior controlo no processo (Huang et al., 2019; Zang et al., 2020).

No agachamento ou utilização de um banco ou bola de parto, a coluna fica ereta e as coxas abduzidas, ajudando a abrir a pelve ao máximo, direcionando o peso para baixo no sacro (Huang et al., 2019). A sínfise púbica e as articulações sacroilíacas são projetadas para fora junto com o movimento para trás do sacro e do cóccix (Desseauve et al., 2019). Assim, 0 agachamento resulta num aumento de $28 \%$ no tamanho da saída pélvica (Huang et al., 2019; Musie et al., 2019).

Na posição de sentada a parturiente aproveita ao máximo as vantagens ergonómicas da gravidade e alivia a dor lombar e dos membros inferiores, além de a ajudar a mover livremente os membros inferiores e conferir boa visibilidade do períneo (Huang et al., 2019; Zang et al., 2020).

Por fim, a posição ajoelhada ou de quatro apoios ajuda a aliviar a tensão nas costas e a aumentar a eficácia dos esforços expulsivos no segundo período do trabalho de parto, apesar de muitas mulheres não conseguirem adotar esta posição por muito tempo (Huang et al., 2019; Zang et al., 2020).

A verticalização do parto reduz significativamente a duração média do primeiro período do trabalho de parto (em 2 horas a menos) e está associada à não necessidade de analgesia epidural, assim como a uma menor probabilidade de parto por cesariana (Gams et al., 2019). No entanto, quando é administrada analgesia por via epidural, a adoção de posições verticais, pode ser largamente desvantajosa em relação à adoção de posições supinas (Brocklehurst, 2017). 


\section{BENEFíCIOS DA VERTICALIZAÇÃo dO PARTO}

Por último, verifica-se a importância dos profissionais de saúde na assistência ao parto, que desempenham um papel fundamental no cuidado e apoio às mulheres durante 0 trabalho de parto, pelo que devem dominar as técnicas necessárias para ajudar e aconselhar com sucesso as parturientes nas diferentes posições (Huang et al., 2019).

\section{CONCLUSÕES}

Nesta revisão integrativa foram explorados os benefícios da verticalização do parto, tendo como princípios a promoção do bem-estar materno, a promoção do bem-estar fetal e a facilitação da progressão do trabalho de parto. Neste sentido, a conceção e prestação de cuidados à parturiente deverá atender a esses princípios, sendo o parto vertical um meio para os atingir. Nos programas de preparação para o parto e nas consultas pré-natais os profissionais de saúde devem sensibilizar a mulher/casal para a adoção dessas posições, bem como proceder à sua capacitação através da transmissão de conhecimentos e técnicas práticas necessários à realização do parto em posição vertical.

É inquestionável que, a adoção de posições verticais no trabalho de parto transmite uma sensação de normalidade, naturalidade, autonomia e controlo à parturiente. Na verdade, várias posições podem e devem ser adotadas durante 0 trabalho de parto e parto, pelos efeitos inerentes na promoção de resultados positivos maternos e neonatais, sendo um direito da parturiente escolher qual ou quais as posições que quer adotar.

Todos os profissionais de saúde desempenham um papel fundamental no cuidado e apoio às mulheres durante o trabalho de parto, sendo importante integrar, em todo o processo que envolve um trabalho de parto, as preocupações e os desejos da mulher, incluindo a posição em que o bebé nasce.

Requere-se que os profissionais de saúde sejam dotados de habilidades e técnicas necessárias para aplicar diferentes posições de acordo com a necessidade e conforto da mulher, sem com isso causar constrangimentos ou perigo para a saúde tanto da parturiente como do feto. É necessário que haja, nas maternidades, um crescente investimento em saberes técnico-científicos sobre os benefícios do parto vertical e condições físicas para que 0 mesmo seja concretizável. É urgente identificar práticas baseadas em evidências que contribuam para o bem-estar materno-fetal e para a humanização do parto.

Concluindo, os benefícios da verticalização do trabalho de parto, promovem o bem-estar fetal, trazem ganhos em saúde para a mulher, ganhos económicos para as unidades hospitalares e experiências de parto mais ativas, agradáveis, felizes e humanizadas.

Uma das limitações incidiu sobre a generalidade da temática e na diversidade de estudos, uma vez que a questão de investigação proposta era bastante abrangente. Um dos aspetos observados durante a pesquisa terá sido a falta de apresentação de evidências e factos da adoção de posições verticais durante o parto, sendo que muitos estudos mantiveram a índole sugestiva e sem provas. Outra limitação observada, foi a falta de estudos, nos idiomas Inglês e Português, que respondam efetivamente à questão proposta nos anos em que incidiu esta revisão (de 2017 a 2020), 0 que reflete uma necessidade de produção de investigação e conhecimento científico nesta área, para uma prática baseada em evidências.

Por último, verificou-se que alguns estudos poderão não refletir o contexto e a realidade da prática dos enfermeiros especialistas em Enfermagem de Saúde Materna e Obstétrica em Portugal, pelo que se sugere mais estudos nas maternidades portuguesas relacionados com o parto vertical.

\section{FINANCIAMENTO E AGRADECIMENTOS}

Este trabalho é financiado por Fundos Nacionais através da FCT - Fundação para a Ciência e a Tecnologia, I.P., no âmbito do projeto Ref ${ }^{\mathfrak{a}}$ UIDB/00742/2020. Agradecemos adicionalmente ao Politécnico de Viseu pelo apoio prestado e à UICISA:E 


\section{REFERÊNCIAS BIBLIOGRÁFICAS}

Ayres, L, Henriques B., \& Amorim, W. (2018). A representação cultural de um "parto natural": 0 ordenamento do corpo grávido em meados do século XX. Ciência \& Saúde Coletiva, 23(11):3525-3534. https://doi.org/10.1590/1413-812320182311.27812016

Bick, D., Briley, A., Brocklehurst, P., Hardy, P., Juszczak, E., Lynch, L., Wilson, M. (2017). A multicentre, randomised controlled trial of position during the late stages of labour in nulliparous women with an epidural: Clinical effectiveness and an economic evaluation (BUMPES). Health Technology Assessment, 21(65), 1175. https://doi.org/10.3310/hta21650

Botelho, L., Cunha, C., \& Macedo, M. (2011). 0 método da revisão integrativa nos estudos organizacionais. Gestão e Sociedade, 5(11), 121-136. https://doi.org/10.21171/ges.v5i11.1220

Brocklehurst, P. (2017). Upright versus lying down position in second stage of labour in nulliparous women with low dose epidural: BUMPES randomised controlled trial. BMJ (Online), 359. https://doi.org/10.1136/bmj.j4471

Desseauve, D., Fradet, L., Lacouture, P., \& Pierre, F. (2019). Is there an impact of feet position on squatting birth position? An innovative biomechanical pilot study. BMC Pregnancy and Childbirth, 19(1). https://doi.org/10.1186/s12884-019-2408-2

DiFranco, J. T., \& Curl, M. (2014). Healthy Birth Practice \#5: Avoid Giving Birth on Your Back and Follow Your Body's Urge to Push. The Journal of Perinatal Education, 23(4), 207-210. https://doi.org/10.1891/10581243.23.4.207

Dundes, L. (1987). The evolution of maternal birthing position. American Journal of Public Health, 77(5), 636641. https://doi.org/10.2105/AJPH.77.5.636

Gams, B., Neerland, C., \& Kennedy, S. (2019). Reducing Primary Cesareans: An Innovative Multipronged Approach to Supporting Physiologic Labor and Vaginal Birth. Journal of Perinatal and Neonatal Nursing, 33(1), 52-60. https://doi.org/10.1097/JPN.0000000000000378

Gião, C.; Pinhão, R. (2012). Mapear o cuidado para regressar a casa: A Qualidade da Intervenção Educativa de Enfermagem no Planeamento da Alta da Pessoa Submetida a Transplante de Progenitores Hematopoiéticos (Dissertação de mestrado). Obtido de https://comum.rcaap.pt/bitstream/10400.26/5475/1/Dissertaca0\%20mapear\%200\%20cuidado\%20para\%2 Oregressar\%20a\%20casa\%20de\%20claudia\%20pinhao.pdf

Gizzo, S., Di Gangi, S., Noventa, M., Bacile, V., Zambon, A., \& Nardelli, G. B. (2014). Women's choice of positions during labour: Return to the past or a modern way to give birth? A cohort study in Italy. BioMed Research International, 2014. https://doi.org/10.1155/2014/638093

Gupta, J. K., Sood, A., Hofmeyr, G. J., \& Vogel, J. P. (2017). Position in the second stage of labour for women without epidural anaesthesia. Cochrane Database of Systematic Reviews. John Wiley and Sons Ltd. https://doi.org/10.1002/14651858.CD002006.pub4

Huang, J., Zang, Y., Ren, L. H., Li, F. J., \& Lu, H. (2019). A review and comparison of common maternal positions during the second-stage of labor. International Journal of Nursing Sciences. Chinese Nursing Association. https://doi.org/10.1016/j.ijnss.2019.06.007

Lin, Y. C., Gau, M. L., Kao, G. H., \& Lee, H. C. (2018). Efficacy of an Ergonomic Ankle Support Aid for Squatting Position in Improving Pushing Skills and Birth Outcomes during the Second Stage of Labor: A Randomized Controlled Trial. Journal of Nursing Research, 26(6), 376-384. https://doi.org/10.1097/jnr.0000000000000262

Louwen, F., Daviss, B. A., Johnson, K. C., \& Reitter, A. (2017). Does breech delivery in an upright position instead of on the back improve outcomes and avoid cesareans? International Journal of Gynecology and Obstetrics, 136(2), 151-161. https://doi.org/10.1002/ijgo.12033

Mineiro, A. (2016). A Posição Da Mulher No Trabalho De Parto. in Néné, M, Marques, R \& Batista, M. (2016). Enfermagem de Saúde Materna e Obstétrica. Lidel, Lisboa, Portugal. 


\section{BENEFÍCIOS DA VERTICALIZAÇÃO dO PARTO}

Miquelutti, M. A., Cecatti, J. G., Morais, S. S., \& Makuch, M. Y. (2009). Posição vertical durante 0 trabalho de parto: Dor e satisfação. Revista Brasileira de Saude Materno Infantil, 9(4), 393-398. https://doi.org/10.1590/S1519-38292009000400002

Musie, M. R., Peu, M. D., \& Bhana-Pema, V. (2019). Factors hindering midwives' utilisation of alternative birth positions during labour in a selected public hospital. African Journal of Primary Health Care and Family Medicine, 11(1). https://doi.org/10.4102/phcfm.v11i1.2071

Nilsen, E., Sabatino, H., \& Lopes, M. (2011). Dor e comportamento de mulheres durante 0 trabalho de parto e parto em diferentes posições. Rev Esc Enferm UPS 45(3):557-65. https://doi.org/10.1590/S008062342011000300002

Organização Mundial de Saúde. (1996). Assistência ao parto normal: Um guia prático. Genebra: Organização mundial de Saúde.

Peters et al. Chapter 11: Scoping Reviews (2020 version). In: Aromataris E, Munn Z (Editors). JBI Manual for Evidence Synthesis, JBI, 2020. Acedido em https://synthesismanual.jbi.global. Doi:10.46658/JBIMES-20-12

Santos, C. M. D. C., Pimenta, C. A. D. M., \& Nobre, M. R. C. (2007). A estratégia PICO para a construção da pergunta de pesquisa e busca de evidências. Revista Latino-Americana de Enfermagem. Associacao Medica Brasileira. https://doi.org/10.1590/S0104-11692007000300023

Simarro, M., Espinosa, J., Salinas, C., Ojea, R., Salvadores, P., Walker, C., \& Schneider, J. (2017). A Prospective Randomized Trial of Postural Changes vs Passive Supine Lying during the Second Stage of Labor under Epidural Analgesia. Medical Sciences, 5(1), 5. https://doi.org/10.3390/medsci5010005

Sousa, J. L., da Silva, I. P., Gonçalves, L. R. R., Nery, I. S., Gomes, I. S., \& Sousa, L. F. C. (2018). Perception of puerperas on the vertical position in childbirth. Revista Baiana de Enfermagem, 32. https://doi.org/10.18471/rbe.v32.27499

WHO. (2018). WHO Recommendations: intrapartum care for a positive childbirth experience. WHO. Retrieved from http://www.who.int/reproductivehealth/publications/intrapartum-care-guidelines/en/

Zang, Y., Lu, H., Zhang, H., Huang, J., Ren, L., \& Li, C. (2020). Effects of upright positions during the second stage of labour for women without epidural analgesia: A meta-analysis. Journal of Advanced Nursing. Blackwell Publishing Ltd. https://doi.org/10.1111/jan.14587 